\title{
Simulation and experiment on prediction of total rice seeds mass in vibrating tray for vacuum plate seeder
}

\author{
Zhan Zhao*, Mingzhi Jin, Chunjie Tian, Fang Qin \\ (Institute of Agriculture Engineering, Jiangsu University, Zhenjiang 212013, Jiangsu, China)
}

\begin{abstract}
To improve the seeds pick-up precision of a vacuum plate seeder, it is important to accurately control the relative distance between suction plate and seeds layer in vibrating tray. Under the excitation of reciprocating vibration with a time varying interference in direction angle, the seeds motion is simulated using the discrete element method (DEM). By analyzing the seeds distribution characteristics, it was found that the seeds-mass-per-unit-area (SMA) was approximately plane distributed in the tray. Then, four square areas on the bottom of rectangular tray were divided symmetrically near the four vertices to measure the corresponding SMA respectively, and a monitoring plane model was established to predict the average SMA and total seeds mass in the tray. The prediction results of DEM simulation showed that the maximum and the mean relative errors were $6.75 \%$ and $2.85 \%$. The influences of the normal vector of monitoring plane model and the standard deviation of SMA in four monitoring areas on the prediction errors were analyzed. A method for improving prediction accuracy by using linear regression correction was proposed, and the maximum and the mean relative errors could be reduced to $5.01 \%$ and $2.07 \%$. When the tray was vibrated with the frequency of $11 \mathrm{~Hz}$ and the amplitude of $4 \mathrm{~mm}$, experiments were carried out on the vacuum plate seeder test-rig. A Kalman filter was adopted to suppress the SMA measurement noise in four monitoring areas. Prediction results indicated that the maximum and the mean relative errors were $10.2 \%$ and $3.46 \%$ with the average SMA in range of $0.9-1.5 \mathrm{~g} / \mathrm{cm}^{2}$, respectively. The paper can provide a basis for further study on the automatic control of suction plate motion according to the variation of seeds mass in the tray.
\end{abstract}

Keywords: vacuum plate seeder, rice seeds, total mass, prediction method, vibrating tray, discrete element method DOI: $10.25165 /$ j.ijabe.20191205.4415

Citation: Zhao Z, Jin M Z, Tian C J, Qin F. Simulation and experiment on prediction of total rice seeds mass in vibrating tray for vacuum plate seeder. Int J Agric \& Biol Eng, 2019; 12(5): 81-86.

\section{Introduction}

Rice is one of the major staple foods in the world, and nursery-transplanting is the mainly used planting pattern. The quality of seedling directly affects the growth and yield of rice after transplanting. With the improvement of rice varieties and the rapid extension of hybrid-rice cultivation, there is a need to improve the seeding precision in the nursery process ${ }^{[1-3]}$. Seeder is the main equipment to perform seeding operation, and many new seeders have been developed in recent years ${ }^{[4-6]}$. Rice seed has the properties of irregular shape, rough surface and large friction coefficient. Existing mechanical metering devices cannot meet its precision seeding agronomic requirements, and damaged seeds are a serious problem. Compared with mechanical seeders, those using vacuum principle are widely used because they have the advantages of more precise seeding quality, lower rate of seeds damage, and broader spectrum of applicability ${ }^{[7-12]}$.

The vacuum plate precision seeder is primary composed of a suction plate and a seeds tray ${ }^{[13]}$. The tray is vibrated at high frequency and small amplitude, so that the seeds in the tray can be

Received date: 2018-05-20 Accepted date: 2019-07-29

Biographies: Mingzhi Jin, Graduate student, research interests: modern agricultural equipment, Email: 347248798@qq.com; Chunjie Tian, MS, research interests: modern agricultural equipment, Email: 779642278@qq.com; Fang Qin, Graduate student, research interests: modern agricultural equipment, Email: 1533286817@qq.com;

*Corresponding author: Zhan Zhao, PhD, Researcher, research interests: monitoring and control technology on agricultural machinery. Institute of Agriculture Engineering, Jiangsu University, Zhenjiang 212013, China. Tel: +86-13852988080, Email: zhaozhan@ujs.edu.cn. separated to reduce interaction forces. According to the structure of seedling nursery tray, multiple nozzles need to be processed on the panel of suction plate. When the plate moves above the vibrating tray, seeds are picked up to the nozzles under the action of the vacuum air flow. Then, the plate moves to the specified position above the nursery tray, and the vacuum switches to positive pressure. Seeds fall onto the nursery tray under the forces of gravity and positive differential pressure.

Recently, a lot of research has been carried out on the structure optimization of seeders, simulation of the airflow field distribution, calculation of the suction force on seeds and working performance experiments ${ }^{[14-17]}$. On the test-rig used in this paper, seeds pick-up performance experiments have been carried out ${ }^{[18,19]}$. Results indicated that the key factor to maintain a good continuous working performance is to keep a proper vertical distance between suction plate and vibrating seeds. During the seeding operation, seeds mass in the tray is continuously changing ${ }^{[20]}$. Therefore, the pickup position of suction plate needs to be adjusted accordingly. For achieving this task, an important challenge to monitor the total seeds mass in the tray real time.

In our previous work ${ }^{[21]}$, the seeds impact mechanical properties under the excitation of vertical reciprocating vibration were analyzed, and a monitoring method and the corresponding device of seeds mass in a small area was proposed. In this paper, seeds motion and distribution variation in a rectangle vibrating tray was simulated by DEM, especially under the vibration with a time varying interference in direction angle. Then, a prediction method of total seeds mass utilizing plane fitting modeling, linear regression correction and Kalman filtering processing was discussed. Finally, experiments were carried out in laboratory to 
evaluate the measurement accuracy.

\section{DEM simulation of seeds motion}

Discrete element method (DEM) proposed by Cundall is the most effective numerical tool for simulating the mechanical, static and dynamic behavior of granular materials. Newton's second motion law and Euler's dynamics equation are usually used to describe the translational and rotational movements of particle, and the overall system behavior can be determined as a result of individual particle interactions. DEM has been successfully applied to the analysis of agricultural particle material motion system $^{[22-25]}$

Seeds are always in motion in the vibrating tray. DEM simulations can provide detailed information, such as the trajectories of and transient forces acting on individual particles, which is extremely difficult to obtain by physical experimentation. Therefore, in this paper, a three-dimensional DEM code $\left(\mathrm{EDEM}^{\mathbb{B}}\right.$ 2.5, DEM Solutions) is used to simulate seeds motions. The mechanism of vibrating tray is shown in Figure 1. Four guiding axes were vertically mounted on an adjustment plate. Driven by a motor and crank mechanism, the tray reciprocated along guiding axes. By adjusting a set of parallel mechanisms, the vibration direction could be rotated along $X$ and $Y$ axes within the angle of $\pm 10^{\circ}$.

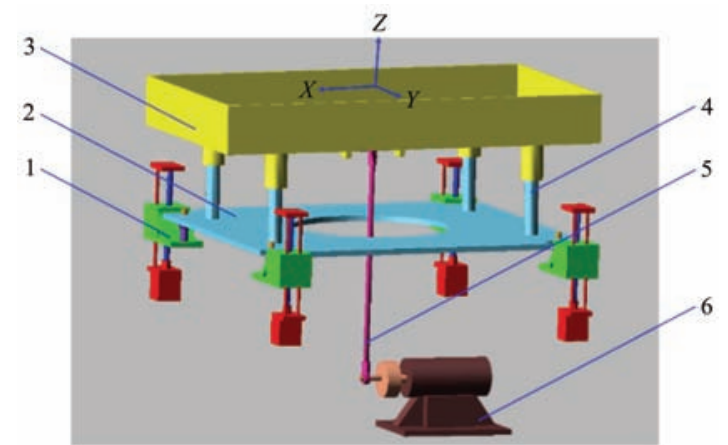

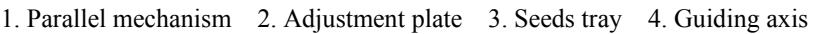
5. Crank mechanism 6. Motor

Figure 1 Mechanism diagram of the vibrating tray

The model of rectangular seeds tray is shown in Figure 2. Its size was $700 \mathrm{~mm} \times 420 \mathrm{~mm}$, and the material was aluminum alloy \#7075. Taking the center of the tray as the origin, the inertial coordinate $\mathbf{X}_{\mathbf{0}}=\left[\begin{array}{lll}X & Y Z\end{array}\right]$ was established.

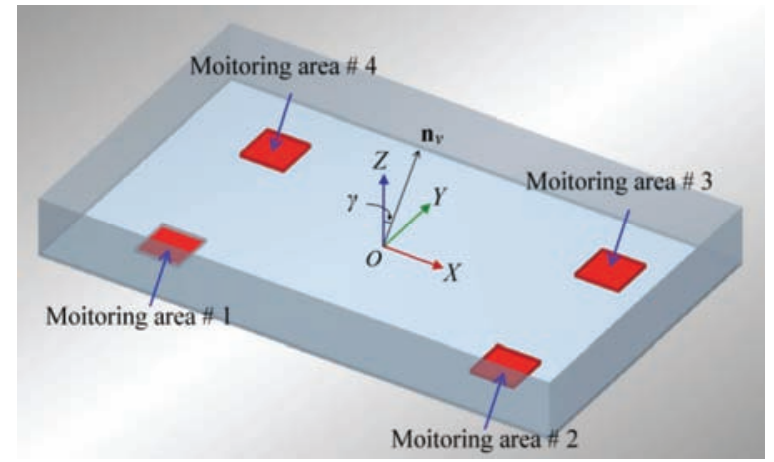

Figure 2 DEM simulation model of seeds tray

Due to the interferences such as installation precision and horizontal posture of the frame, the tray can not always keep vertical vibration along the $Z$-axis. There is usually an uncertain small time varying angle $\gamma$ between the vibration direction vector $\mathbf{n}_{\mathrm{v}}$ and the $Z$-axis.

Many experimental results have indicated that, to improve the seeding performance, the seeds layer thickness in the vibrating tray should be maintained in a reasonable range. For rice seeds seeding, this range is general between $15-25 \mathrm{~mm}$, which corresponding to the seeds-mass-per-unit-area (SMA) is about $0.9-1.5 \mathrm{~g} / \mathrm{cm}^{2}$. And the ideal vibration frequency $f$ and amplitude $A$ of tray are near $11 \mathrm{~Hz}$ and $4 \mathrm{~mm}^{[13,26]}$. Therefore, DEM simulations are performed under the vibration parameters, and three average SMA $\kappa_{0}$ of $0.9 \mathrm{~g} / \mathrm{cm}^{2}, 1.2 \mathrm{~g} / \mathrm{cm}^{2}$ and $1.5 \mathrm{~g} / \mathrm{cm}^{2}$ are selected.

The Hertz-Mindlin model is used to calculate contact force ${ }^{[27,28]}$. The normal force $F_{\mathrm{n}}$ and tangential force $F_{\tau}$ can be calculated by

$$
\begin{gathered}
F_{n}=\frac{4}{3} E_{0} \delta_{n}^{\frac{3}{2}} \sqrt{R_{0}}-2 \sqrt{\frac{5}{6}} \frac{\ln e}{\sqrt{\ln ^{2} e+\pi^{2}}} \sqrt{2 E_{0}} \sqrt[4]{R_{0} \delta_{n}} \sqrt{m_{0}} v_{n}^{r e l} \\
F_{\tau}=-8 G_{0} \sqrt{R_{0} \delta_{n} \delta_{\tau}}-2 \sqrt{\frac{5}{6}} \frac{\ln e}{\sqrt{\ln ^{2} e+\pi^{2}}} \sqrt{2 E_{0}} \sqrt[4]{R_{0} \delta_{n}} \sqrt{m_{0}} v_{\tau}^{r e l}
\end{gathered}
$$

where, $E_{0}$ is the equivalent Young's modulus of the two interacting particles, MPa; $\delta_{\mathrm{n}}$ is the normal overlap, $\mathrm{m} ; R_{0}$ is the equivalent radius, $\mathrm{m} ; m_{0}$ is the equivalent mass, $\mathrm{kg} ; e$ is the coefficient of restitution; $v_{\mathrm{n}}{ }^{\text {rel }}$ is the normal relative velocity, $\mathrm{m} / \mathrm{s} ; G_{0}$ is the equivalent shear modulus of the two interacting particles, MPa; $\delta_{\tau}$ is the tangential overlap, $\mathrm{m} ; v_{\tau}{ }^{\text {rel }}$ is the tangential relative velocity, $\mathrm{m} / \mathrm{s}$.

The proposed monitoring method of seeds mass in a small area indicated that the effect of grain shape on measurement error is slight ${ }^{[21]}$. According to the physical properties of rice seeds Changyou \#5 used in experiments, a triaxial ellipsoidal particle model was established. Semi-axes of the model were $2.85 \mathrm{~mm}$, $1.55 \mathrm{~mm}$ and $1.35 \mathrm{~mm}$, and the mass of single seed was $26.5 \times 10^{-3} \mathrm{~g}$. The density was $1080 \mathrm{~kg} / \mathrm{m}^{3}$, Young's modulus was $375 \mathrm{MPa}$, Poisson's ratio was 0.25 , coefficients of friction and restitution between seeds were 0.48 and 0.42 , coefficients of friction and restitution between seeds and plate were 0.32 and $0.48^{[21,29]}$. A time varying interference angle $\gamma$ within the range of $\pm 2.5^{\circ}$ was applied randomly in the vibration direction vector $\mathbf{n}_{\mathrm{v}}$ to make the seeds flow in the tray. The DEM simulation of seeds motion state is shown in Figure 3.

To analyze the seeds distribution, the tray was divided into $10 \times 6$ small areas. By counting the number of seeds, the seeds mass in each area could be calculated at each moment. Then, according to the central coordinates of all areas and the corresponding seeds mass, the actual seeds distribution model could be established using multiple regressions.

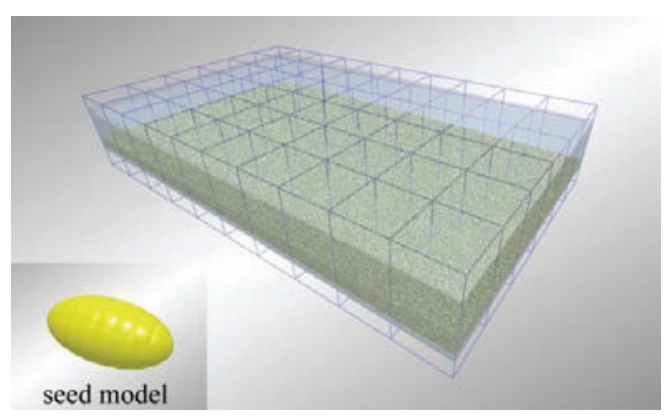

Figure 3 DEM simulation of seeds motion

It is found that, under the excitation of reciprocating vibration, the seeds distribution can be approximately fitted using a plane model due to the bottom of the tray is flat. The fitting error majorly occurs in the marginal area of the tray. Regression analysis results show that the overall root mean squared errors (RMSE) are less than $2.5 \mathrm{~mm}$ and the coefficients of determination 
are greater than 0.9 . With the average SMA $\kappa_{0}$ of $0.9 \mathrm{~g} / \mathrm{cm}^{2}$, $1.2 \mathrm{~g} / \mathrm{cm}^{2}$ and $1.5 \mathrm{~g} / \mathrm{cm}^{2}$, select data at some time points from the three DEM simulations and the corresponding fitting results are shown in Figure 4.

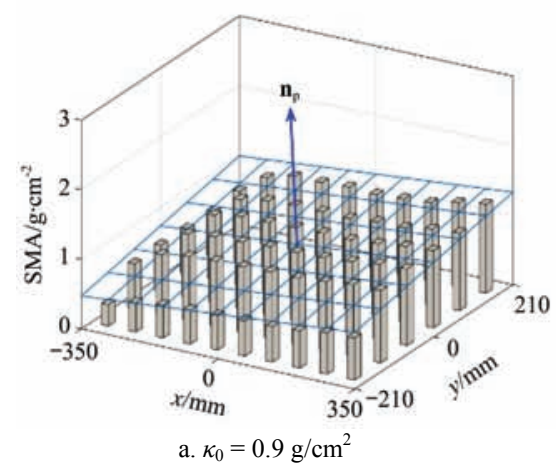

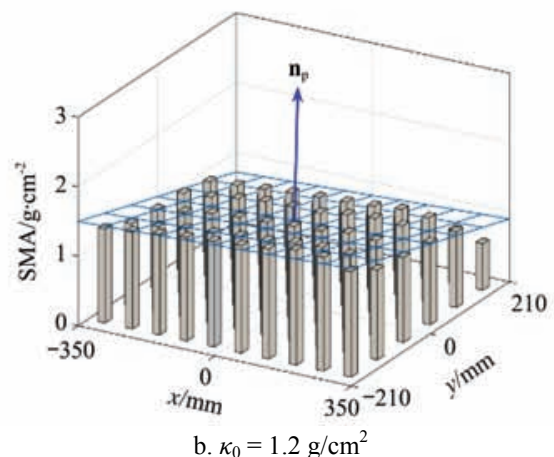

b. $\kappa_{0}=1.2 \mathrm{~g} / \mathrm{cm}^{2}$

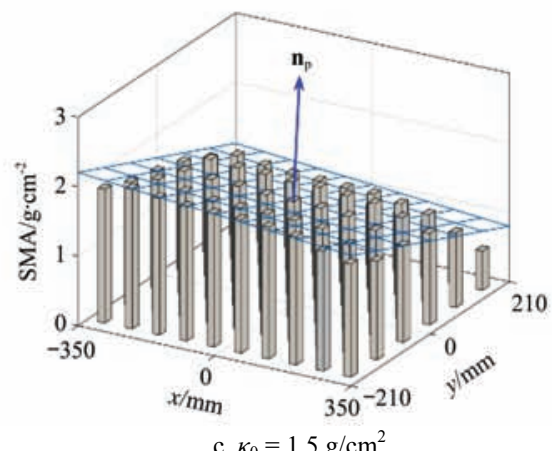

c. $\kappa_{0}=1.5 \mathrm{~g} / \mathrm{cm}^{2}$

Figure 4 Plane fitting results of seeds distribution

\section{Prediction method}

The total seeds mass in the tray $M$ can be calculated as the product of average SMA $\kappa_{0}$ and tray area $S, M=S \times \kappa_{0}$. So, the total seeds mass measurement is converted to the prediction of average SMA $\kappa_{0}$. Assume the plane fitting model equation $\mathrm{P}$ of seeds distribution is given by

$$
\mathrm{P}: A x+B y+C z+D=0
$$

The normal vector of the plane $\mathbf{n}_{\mathrm{p}}$ is $[A, B, C]$. Obviously, the value of $z$ at the center point of the plane $\mathrm{P}(x=0, y=0)$ directly reflects the value of $\kappa_{0}$, which is denotes as $\kappa_{\mathrm{p}}$ and can be calculated by

$$
\kappa_{\mathrm{p}}=-D / C
$$

DEM simulation results indicate that the difference between $\kappa_{\mathrm{p}}$ and $\kappa_{0}$ during the whole process is less than $0.4 \%$. Therefore, this is a feasible way for the prediction of $\kappa_{0}$. In addition, the seeds distribution model can also provide a basis for the adjustment of the vibrating tray to obtain a reasonable seeds distribution ${ }^{[26]}$.

However, during the actual operation of the seeder, it is impossible to count the number of seeds, and it is also difficult to measure SMA of in many areas. A plane model can be obtained by SMA in three regions. With the increasing of the number of monitoring area and the distance between multiple monitoring areas, the stability of the fitting model will be improved. The plane fitting results of seeds distribution indicated that the error majorly occurs in the marginal area of the tray, and the randomness of the seeds number is relatively large. Therefore, in order to

reduce this effect, four $60 \mathrm{~mm} \times 60 \mathrm{~mm}$ square areas on the bottom were divided symmetrically near the four vertices of the tray for SMA monitoring. The distances between the center of each monitoring area and the corresponding vertex in $X$-axis and $Y$-axis directions were 120 and $95 \mathrm{~mm}$, shown in Figure 2. During the simulations, impact forces of seeds on the four monitoring areas were recorded. Then, a signal calculation majorly integrated a low pass filter was adopted to obtained the stable SMA. The transmissibility equation is given by

$$
H(s)=\frac{A_{0} \omega_{c}^{2}}{s^{2}+\sqrt{2} \omega_{c} s+\omega_{c}^{2}}
$$

where, $\omega_{\mathrm{c}}$ is the critical angular frequency, $\mathrm{rad} / \mathrm{s} ; A_{0}$ is the transmission gain.

The critical frequency of filter was set as $1 \mathrm{~Hz}$. According to the linear relationship between the impact force after filtering and the SMA, the received variations of SMA in four monitoring areas are shown in Figure 5. Using the central coordinates of monitoring areas $\left[x_{1}, y_{1}\right],\left[x_{2}, y_{2}\right],\left[x_{3}, y_{3}\right],\left[x_{4}, y_{4}\right]$ and the corresponding SMA $\kappa_{1}, \kappa_{2}, \kappa_{3}$ and $\kappa_{4}$, a monitoring plane model $\mathrm{P}_{\mathrm{m}}$ can be established using multiple linear regression, which is given by

$$
\mathrm{P}_{\mathrm{m}}: A_{\mathrm{m}} x+B_{\mathrm{m}} y+C_{\mathrm{m}} z+D_{\mathrm{m}}=0
$$

The normal vector of the plane $\mathbf{n}_{\mathrm{m}}$ is $\left[A_{\mathrm{m}}, B_{\mathrm{m}}, C_{\mathrm{m}}\right]$, and the value of $z$ at the center point of the plane $\mathrm{P}_{\mathrm{m}}$ is denoted as $\kappa_{\mathrm{m}}$, which can be calculated by

$$
\kappa_{\mathrm{m}}=-D_{\mathrm{m}} / C_{\mathrm{m}}
$$

The paper proposes to predict the average SMA $\kappa_{0}$ using the value of $\kappa_{\mathrm{m}}$.
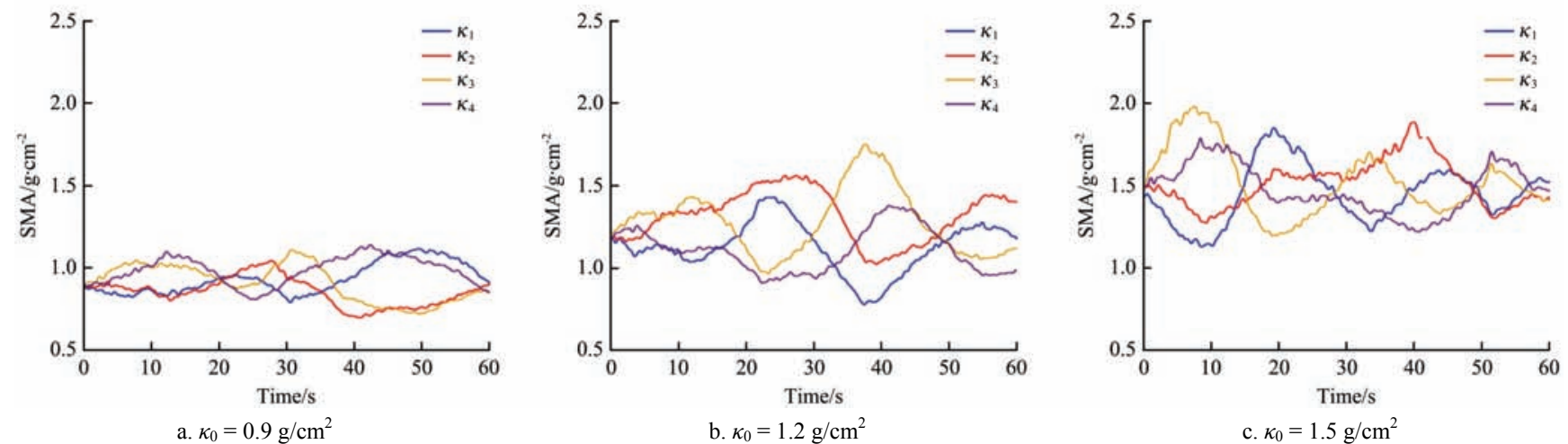

Figure 5 Simulation results of SMA in four monitoring areas

\section{Results and discussion}

\subsection{Prediction results}

According to the DEM simulation results of $\kappa_{1}, \kappa_{2}, \kappa_{3}$ and $\kappa_{4}$, which are given in Figure 5, the prediction of $\kappa_{\mathrm{m}}$ calculated using
Equation (7) are shown in Figure 6. It is obvious that $\kappa_{\mathrm{m}}$ can generally reflect the variation of average SMA $\kappa_{0}$. Under the influence of vibration interference angle, the seeds will flow in the tray and result in an uneven distribution. The seeds distribution surface is no longer a perfect plane. This lead to a certain 
difference between the monitoring plane $\mathrm{P}_{\mathrm{m}}$ and the actual distribution plane $\mathrm{P}$, and cause the value of $\kappa_{\mathrm{m}}$ to fluctuate within a certain range. The calculation results show that the maximum and the mean values of relative error $E_{\delta}$ between $\kappa_{\mathrm{p}}$ and $\kappa_{0}$ are $6.75 \%$ and $2.85 \%$.

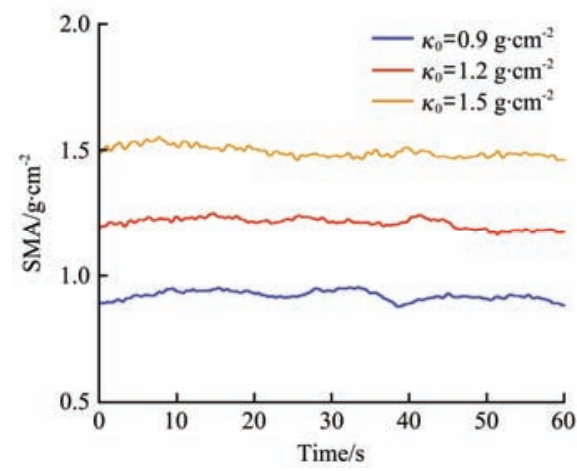

Figure 6 Prediction results of $\kappa_{\mathrm{m}}$ using DEM simulation data

\subsection{Optimized analysis}

The seeds distribution state is an important factor that influences the prediction error. In the paper, the parameters $\varphi$ and $\sigma$ are adopted to reflect the seeds distribution characteristics respectively. The $\varphi$ is defined as the angle between the normal vector of the monitoring plane $\mathbf{n}_{\mathrm{m}}$ and the $Z$ axis, and the $\sigma$ is the standard deviation of $\kappa_{1}, \kappa_{2}, \kappa_{3}$ and $\kappa_{4}$. They can be calculated by

$$
\begin{aligned}
& \cos \varphi=\frac{C_{\mathrm{m}}}{\sqrt{A_{\mathrm{m}}^{2}+B_{\mathrm{m}}^{2}+C_{\mathrm{m}}^{2}}} \\
& \sigma=\sqrt{\frac{1}{4} \sum_{i=1}^{4}\left(\kappa_{i}-\kappa_{0}\right)^{2}}
\end{aligned}
$$

The absolute error between $\kappa_{\mathrm{m}}$ and $\kappa_{0}$ is defined as $\delta_{\mathrm{\kappa}}$, which can be expressed as

$$
\delta_{\kappa}=\kappa_{0}-\kappa_{\mathrm{m}}
$$

After normalizing the value of $\delta_{\mathrm{\kappa}}$, the calculated variations of $\varphi$, $\sigma$ and the corresponding $\delta_{\mathrm{\kappa}}$ are shown in the Figure 7. It is impossible to establish accurate mathematical models due to the randomness of seeds motion. But overall, with the increase of $\varphi$ and $\sigma$, there is a certain decreasing trend of $\delta_{\mathrm{\kappa}}$. Therefore, two linear regression correction equations $\mathrm{L}_{1}$ and $\mathrm{L}_{2}$ are established, they are given by

$$
\begin{aligned}
& \mathrm{L}_{1}: \delta_{\mathrm{K}}=-0.0188 \varphi-0.00859 \\
& \mathrm{~L}_{2}: \delta_{\mathrm{K}}=-0.08 \sigma-0.00206
\end{aligned}
$$

The error analysis results of $E_{\varphi}$ and $E_{\sigma}$ are given in Table 1 . $E_{\varphi}$ and $E_{\sigma}$ are the relative errors between $\kappa_{\mathrm{p}}$ and $\kappa_{0}$ after correction using $\mathrm{L}_{1}$ and $\mathrm{L}_{2}$, and their values are both reduced after the correction. When the seeds layer thickness is smaller, the randomness of seeds motion is stronger, which leads to the larger error in the prediction. With the increasing of $\kappa_{0}$, the error decreases gradually. By comparation, the prediction precision using standard deviation correction equation $\mathrm{L}_{2}$ is higher. The maximum and the mean values of relative errors $E_{\sigma}$ can be controlled below $5.01 \%$ and $2.07 \%$.
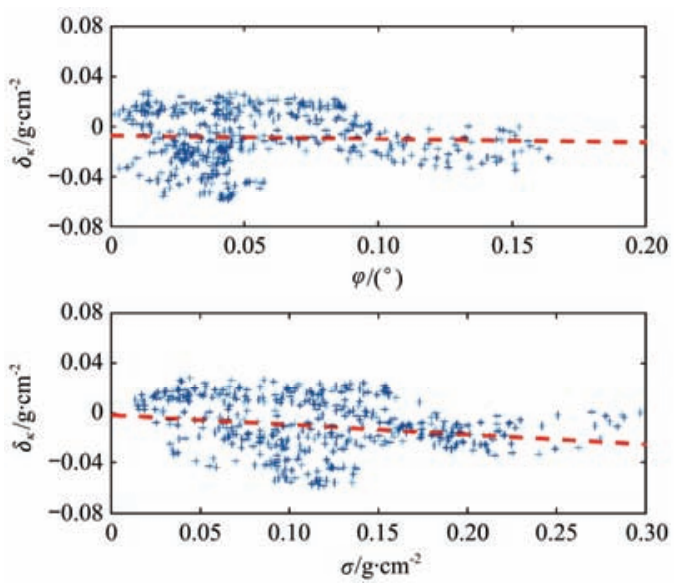

Figure 7 Variations of $\delta_{\kappa}$ versus $\varphi$ and $\sigma$

\section{Verification experiments}

\subsection{Experiment method}

The vacuum plate seeder used in experiments is shown in Figure 8 . The size of rectangular seeds tray was $700 \mathrm{~mm} \times 420$ $\mathrm{mm}$. We have designed the SMA monitoring device and the corresponding signal processing circuits $^{[21]}$. With the vibration frequency and amplitude of $11 \mathrm{~Hz}$ and $4 \mathrm{~mm}$, SMA in the range of $0.3-2.4 \mathrm{~g} / \mathrm{cm}^{2}$, the relative measurement error is less than $2.5 \%$. Four sets of monitoring devices were installed on the tray. The installation position is the same as shown in Figure 2, and the output voltages were recorded using a PLC controller system.

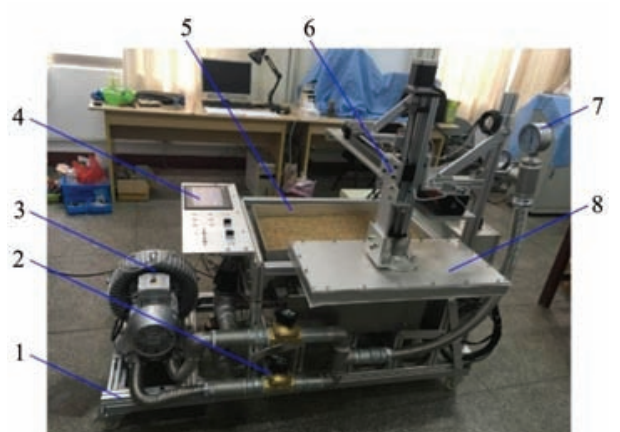

1. Frame 2. Exchange valve 3. Fan 4. PLC controller 5. Seeds tray 6. Cross-sliding table 7. Pressure gauge 8. Suction plate

Figure 8 Precision seeder test-rig using vacuum plate

During the experiments, a certain mass of rice seeds was laid evenly in the tray. Then, the vibration direction $\mathbf{n}_{\mathrm{v}}$ was manually adjusted to make the seeds flow. With the average SMA $\kappa_{0}$ of 0.9 $\mathrm{g} / \mathrm{cm}^{2}, 1.2 \mathrm{~g} / \mathrm{cm}^{2}$ and $1.5 \mathrm{~g} / \mathrm{cm}^{2}$, the measured variations of $\kappa_{1}, \kappa_{2}$, $\kappa_{3}$ and $\kappa_{4}$ are shown in Figure 9.

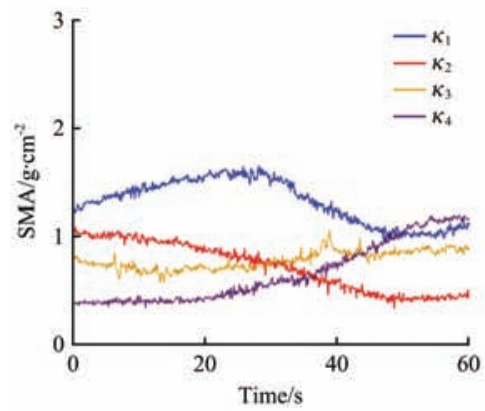

a. $\kappa_{0}=0.9 \mathrm{~g} / \mathrm{cm}^{2}$

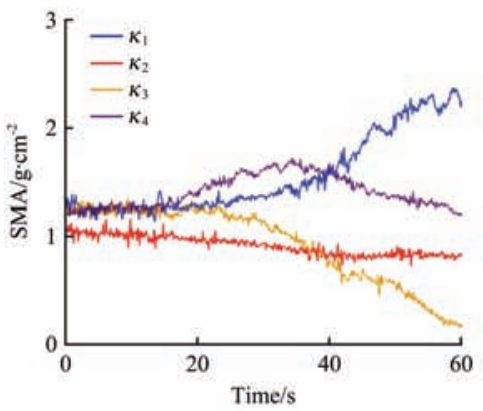

b. $\kappa_{0}=1.2 \mathrm{~g} / \mathrm{cm}^{2}$

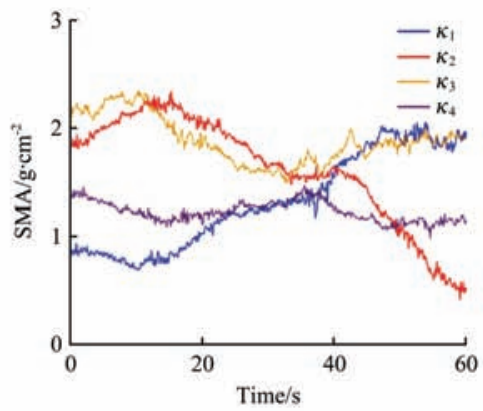

c. $\kappa_{0}=1.5 \mathrm{~g} / \mathrm{cm}^{2}$

Figure 9 Experimental results of SMA in four monitoring areas 


\subsection{Noise reduction using KF}

Measurement noise is inevitable during the experiments, which is mainly caused by complex real environment and device measurement error. Here, a Kalman filter (KF) is adopted to reduce the noise. It aims at producing values closer to the true values of measurements by using the noisy observation. The filter predicts the values by estimating the uncertainty of the predicted value and computing weighted average of the predicted value and measured value. Therefore, the filtering results have less uncertainties $^{[30,31]}$.

Assume the system and measurement equations in discrete form are given by

$$
\begin{gathered}
x_{k}=A x_{k-1}+B u_{k-1}+\omega_{k-1} \\
z_{k}=H_{k} x_{k}+\varepsilon_{k}
\end{gathered}
$$

where, $x$ is the system state; $z$ is the measured output; $u$ is the system input; $\omega$ and $\varepsilon$ are process and measurement noise; $A, B$ and $H$ are state, input and output matrices respectively.

The Kalman filtering is defined in two steps. In the first step, the information from the previous step is incorporated into the filter by generating a predicted state

$$
\begin{gathered}
\hat{x}_{k}^{-}=A \hat{x}_{k-1}+B u_{k} \\
P_{k}^{-}=A P_{k-1} A^{T}+Q
\end{gathered}
$$

where, $Q$ is the model stochastic error covariance; superscript '-' denotes an intermediate step between $k-1$ and $k$.

In the second step, the predicted state is corrected using data from the current state via the measured output $z$ and the predicted matrix $P_{\mathrm{k}}^{-}$. Equations for state estimate and error covariance update can be represented as

$$
\begin{gathered}
\hat{x}_{k}=\hat{x}_{k}^{-}+K_{k}\left(z_{k}-H \hat{x}_{k}^{-}\right) \\
K_{k}=P_{k}^{-} H_{k}^{T}\left(H_{k} P_{k}^{-} H^{T}+R\right)^{-1} \\
P_{k}=P_{k}^{-}-K_{k} H_{k} P_{k}^{-}
\end{gathered}
$$

where, $K_{\mathrm{k}}$ is the Kalman gain; $R$ is the measurement noise variance.

The total seeds mass in the tray is constant in each experiment, and the dimension of SMA equals to one. So, there is $A=1$ and $H=1$. The random noise ratio is set as $2.5 \%$. We filter the measured data sequences $\kappa_{1}, \kappa_{2}, \kappa_{3}$ and $\kappa_{4}$ (shown in Figure 9) through iterative process, and then the monitoring plane model $\mathrm{P}_{\mathrm{m}}$ is established. The calculated prediction values of $\kappa_{\mathrm{m}}$ using Equation (7) are shown in Figure 10.

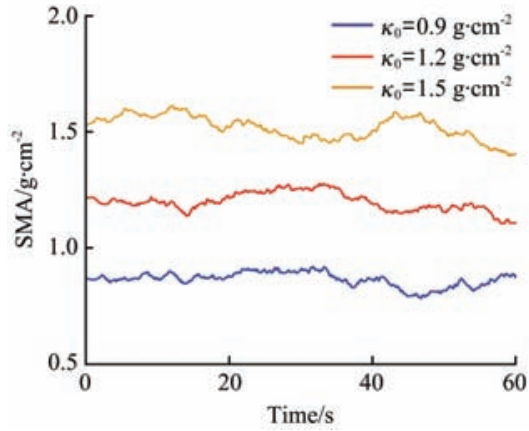

Figure 10 Prediction values of $\kappa_{\mathrm{m}}$ using Kalman filter

\subsection{Results}

It is obviously that the measurement noise is effectively suppressed by using Kalman filter, which can reduce the instantaneous random errors. There are some increasing in the prediction errors, due to the actual disturbance and uncertainty factors are stronger than DEM simulations. Using the same correction method, two linear fitting equations $\mathrm{L}_{3}$ and $\mathrm{L}_{4}$ are established, which can be expressed as

$$
\begin{aligned}
& \mathrm{L}_{3}: \delta_{\kappa}=-0.0153 \varphi+0.0103 \\
& \mathrm{~L}_{4}: \delta_{\kappa}=-0.0553 \sigma-0.0075
\end{aligned}
$$

Through comparison, it can be seen that the correction equations established by DEM simulations and experiments have similarity characteristics, although there are some differences in the fitting coefficients. And the prediction errors can be reduced by using correction equation $\mathrm{L}_{3}$ and $\mathrm{L}_{4}$. The detailed error analysis is given in Table 1.

The seeds distribution in the tray is an important factor affecting the measurement results, since the prediction method is based on the plane fitting model. The seeds flow in the tray is affected by the layer thickness. When the layer thickness is small, the randomness of the flow is strong, which will increase the uncertainty of seeds distribution and result in a larger measurement error. With the increase of layer thickness, the seeds distribution

\begin{tabular}{|c|c|c|c|c|c|c|c|c|c|c|c|c|c|c|}
\hline \multirow{4}{*}{ SMA } & \multirow{2}{*}{\multicolumn{6}{|c|}{ DEM Simulation }} & \multicolumn{8}{|c|}{ Experiment } \\
\hline & & & & & & & \multicolumn{6}{|c|}{ With KF } & \multicolumn{2}{|c|}{ Without KF } \\
\hline & \multicolumn{3}{|c|}{ Maximum } & \multicolumn{3}{|c|}{ Mean } & \multicolumn{3}{|c|}{ Maximum } & \multicolumn{3}{|c|}{ Mean } & \multirow{2}{*}{$\frac{\text { Maximum }}{E_{\delta}}$} & \multirow{2}{*}{$\begin{array}{c}\text { Mean } \\
E_{\delta}\end{array}$} \\
\hline & $E_{\delta}$ & $E_{\varphi}$ & $E_{\sigma}$ & $E_{\delta}$ & $E_{\varphi}$ & $E_{\sigma}$ & $E_{\delta}$ & $E_{\varphi}$ & $E_{\sigma}$ & $E_{\delta}$ & $E_{\varphi}$ & $E_{\sigma}$ & & \\
\hline$\kappa_{0}=0.9 \mathrm{~g} / \mathrm{cm}^{2}$ & 6.75 & 5.21 & 5.01 & 2.85 & 2.16 & 2.07 & 11.6 & 10.4 & 10.2 & 4.07 & 3.49 & 3.24 & 12.8 & 3.95 \\
\hline$\kappa_{0}=1.5 \mathrm{~g} / \mathrm{cm}^{2}$ & 3.29 & 3.66 & 3.37 & 1.33 & 1.69 & 1.47 & 8.85 & 8.70 & 8.52 & 3.62 & 3.44 & 3.46 & 11.1 & 3.79 \\
\hline
\end{tabular}
in the tray gradually becomes stable, and the measurement error generally decreases.

Using the proposed method, the absolute error of seeds layer thickness can be controlled within $2.2 \mathrm{~mm}$. This can provide a basis for the control of pick-up position of the tray, which is important to maintain the continuous working performance of a vacuum plate seeder ${ }^{[16,18,20]}$.

Table 1 Error analysis of prediction results (\%)

\section{Conclusions}

Discrete element method was used to simulate the seeds motion in a rectangular vibrating tray which has a time varying interference in direction angle. According to the seeds distribution characteristics, a plane fitting model of SMA was established. It was deduced that the value of the model at the center point can be used to reflect the average SMA in the tray.
In order to acquire the seeds distribution, four square areas on the bottom of tray were divided symmetrically near the four vertices to measure the corresponding SMA respectively. And a monitoring plane model was established to predict the average SMA. The prediction results of DEM simulations showed that the maximum and the mean relative errors were $6.75 \%$ and $2.85 \%$. By using the linear fitting correction method, the errors reduced to $5.01 \%$ and $2.07 \%$. Seeds total mass prediction experiments were 
carried out on vacuum plate seeder test-rig. By using a Kalman filter to suppress the measurement noises, the maximum and the mean relative prediction errors can be controlled below $10.2 \%$ and $3.46 \%$.

The paper provides an effective way for prediction of total seeds mass in a vibrating tray, which is an important basis for the determination of seeds pickup position of suction plate, and for improving continuous working performance of vacuum plate seeder.

\section{[References]}

[1] Yang Z Y, Li N, Ma J, Sun Y J, Xu H. High-yielding traits of heavy panicle varieties under triangle planting geometry: A new plant spatial configuration for hybrid rice in China. Field Crops Research, 2014; 168: 135-147.

[2] Sun T, Shang W N, Cao H F, Zhang J B, Jin X Y. Effects of different seeding quantity on rice growing and yield. Chinese Agricultural Science Bulletin, 2005; 21(7): 134-137. (in Chinese)

[3] Jiang X L, Li X Y, Chi Z Z, Wang S H, Yang F M, Zheng J G. Research on potted-tray grown rice seedling transplanting by machine. Agricultural Science \& Technology, 2014; 15(11): 1923-1927.

[4] Yi S J, Liu Y F, Wang C, Tao G X. Experimental study on the performance of bowl-tray rice precision seeder. Int J Agric \& Biol Eng, 2014; 7(1): 17-25.

[5] Gaikwad B B, Sirohi N P S. Design of a low-cost pneumatic seeder for nursery plug trays. Biosystems Engineering, 2008; 99: 322-329.

[6] Movahedi E, Rrzvani M, Hemmat A. Design, development and evaluation of a pneumatic seeder for automatic planting of seeds in cellular trays. Journal of Agricultural Machinery, 2016; 4(1): 65-72.

[7] Topakci M, Karayel D, Canakci M, Furat S, Uzun B. Sesame hill dropping performance of a vacuum seeder for different tillage practices. Applied engineering in agriculture, 2011; 27(2): 203-209.

[8] Rathinakumari A C, Kumaran G S, Mandhar S C. Design and development of tray type vacuum seeder and tray type. Applied horticulture; 2005; 7(1): 49-51.

[9] Karayel D. Performance of a modified precision vacuum seeder for no-till sowing of maize and soybean. Soil \& Tillage Research, 2009; 104(1): 121-125.

[10] Liu L J, Yang H, Ma S C. Experimental study on performance of pneumatic seeding system. Int J Agric \& Biol Eng, 2016; 9(6): 84-90.

[11] Masami F, Tadashi C, Yukiharu S, Takayuki T, Masahiro S, Hisashi H. Developing direct seeding cultivation using an air-assisted strip seeder. Japan Agricultural Research Quarterly, 2015; 49(3): 227-233.

[12] He X, Wang Z M, Luo X W, Cao X M, Liu C B, Zang Y. General structure design and field experiment of pneumatic rice direct-seeder. Int J Agric \& Biol Eng, 2017; 10(6): 31-41.

[13] Chen J, Gong Z Q, Li Y M, Li J H, Xu Y. Experimental study on nursing seedlings of super rice precision seeder device. Transactions of the CSAM, 2015; 46(1): 73-78. (in Chinese)

[14] Yazgi A, Degirmencioglu A. Optimisation of the seed spacing uniformity performance of a vacuum-type precision seeder using response surface methodology. Biosystems Engineering, 2007; 97(3): 347-356.

[15] Abdolahzare Z, Asoodar M A, Kazemi N, Rahnama M, Mehdizadeh S A Optimization of the most important operational parameters of a pneumatic seeder using real-time monitoring for Cucumber and Watermelon seeds. Journal of Agricultural Machinery, 2016; 1: 35-48.

[16] Liu C L, Song J N, Wang J C, Wang C. Analysis of flow field simulation on vacuum seed-metering components of precision metering device with sucker. Journal of China Agricultural University, 2014; 45(6): 92-97. (in Chinese)

[17] Gaikwad B B, Sirohi N P S. Design of a low-cost pneumatic seeder for nursery plug trays. Biosystems Engineering, 2008; 99(3): 322-329.

[18] Zhao Z, Tian C J, Huang H D, Yang S X. Optimization of suction plate structure and seed pickup performance for precision nursery seeder. International Agricultural Engineering Journal, 2019; 28(1): 153-161.

[19] Zhao Z, Tian C J, Wu Y F, Huang H D. Dynamic simulation of seed pick-up process and parameter optimization on vacuum plate seeder for rice. Transactions of CSAE, 2018; 34(7): 38-44. (in Chinese)

[20] Chen J, Li J H, Li Y M, Gong Z Q. Analysis of suction height and seed-adding device for suction-vibration precision seeder. Transactions of the CSAM, 2013; 44(1): 67-71. (in Chinese)

[21] Zhao Z, Wu Y F, Yin J J, Tang Z. Monitoring method of rice seeds mass in vibrating tray for vacuum-panel precision seeder. Computers and Electronics in Agriculture, 2015; 114: 25-31.

[22] Tijskens E, Ramon H, Baerdemaeker De J. Discrete element modelling for process simulation in agriculture. Journal of Sound and Vibration, 2003; 266 : 493-514.

[23] Josephine B, Ambrose R, Mark C, Ronaldo M, Dirk M. Applications of discrete element method in modeling of grain postharvest operations. Food Engineering Reviews, 2014; 6(4): 128-149.

[24] Horabik J, Molenda M. Parameters and contact models for DEM simulations of agricultural granular materials: A review. Biosystems Engineering, 2016; 147: 206-225.

[25] Wang J W, Tang H, Wang J F, Li X, Huang H N. Optimization design and experiment on ripple surface type pickup finger of precision maize seed metering device. Int J Agric \& Biol Eng, 2017; 10(1): 61-71.

[26] Zhao Z, Jin M Z, Tian C J, Yang S X. Prediction of seed distribution in rectangular vibrating tray using grey model and artificial neural network. Biosystems Engineering, 2018; 175: 194-205.

[27] Raji A O, Favier J F. Model for the deformation in agricultural and food particulate materials under bulk compressive loading using discrete element method. I: Theory, model development and validation. Journal of Food Engineering, 2004; 64: 359-371.

[28] Li H C, Li Y M, Gao F, Zhao Z, Xu L Z. CFD-DEM simulation of material motion in air-and-screen cleaning device. Computers and Electronics in Agriculture, 2012; 88: 111-119.

[29] Varnamkhasti M G, Mobli H, Jafari A, et al. Some physical properties of rough rice grain. Journal of Cereal Science, 2008; 47(3): 496-501.

[30] Auger F, Hilairet M, Guerrero J M, Monmasson E, Orlowska-Kowalska T, Katsura S. Industrial applications of the Kalman filter: A review. IEEE Transactions on Industrial Electronics, 2013; 60(12): 5458-5471.

[31] Martin B, Nick W. The Kalman filter for linear systems on time scales. Journal of Mathematical Analysis and Applications, 2013; 406: 419-436. 